\title{
Enhancing the Efficiency of GaSb Photovoltaic Cell Using Thin Film Multiscale Haze and Radiative Cooling
}

Prince Gupta ${ }^{12 \S^{*}}$, Yeonhong Kim ${ }^{1 \S}$, Jonghyeok $\operatorname{Im}^{1}$, Gumin $\mathrm{Kang}^{3}$, Augustine M. Urbas ${ }^{4}$, and Kyoungsik Kim ${ }^{1 *}$,

${ }^{1}$ School of Mechanical Engineering, Yonsei University, 50Yonsei-ro, Seodaemun-gu,Seoul 03722, Republic of Korea.

${ }^{2}$ NanoSYD, Mads Clausen Institute, University of Southern Denmark, Alsion 2, 6400 Sønderborg, Denmark.

${ }^{3}$ Nanophotonics Research Center, Korea Institute of Science and Technology (KIST), Seoul 02792, Republic of Korea.

${ }^{4}$ Materials and Manufacturing Directorate, Air Force Research Laboratory, WrightPatterson AFB, Ohio 45433, United State of America.

$\S$ These authors contributed equally.

${ }^{*}$ Correspondence and requests for materials should be addressed to K.K. and P.G. (e-mails: kks@yonsei.ac.kr, prince@mci.sdu.dk). 
a)

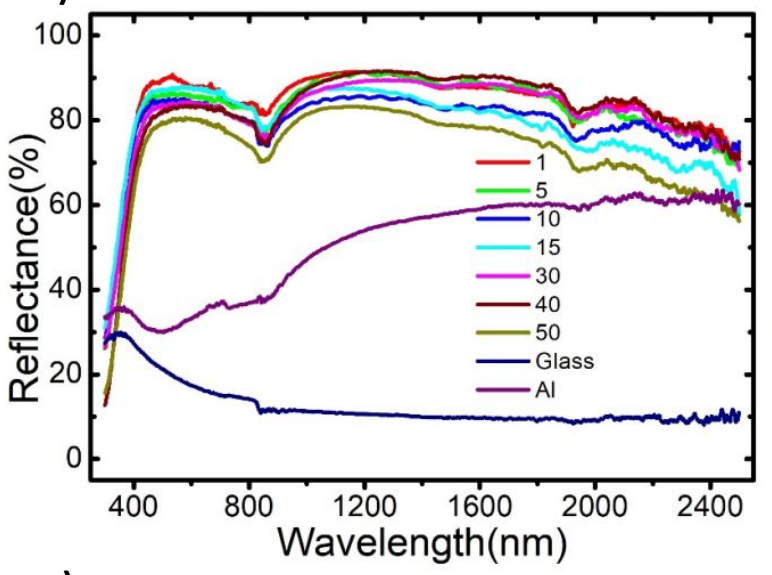

c)

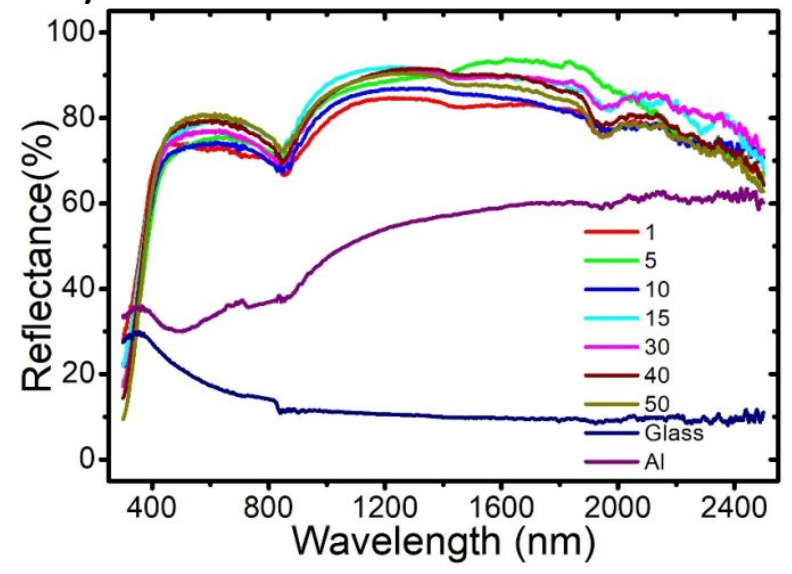

b)

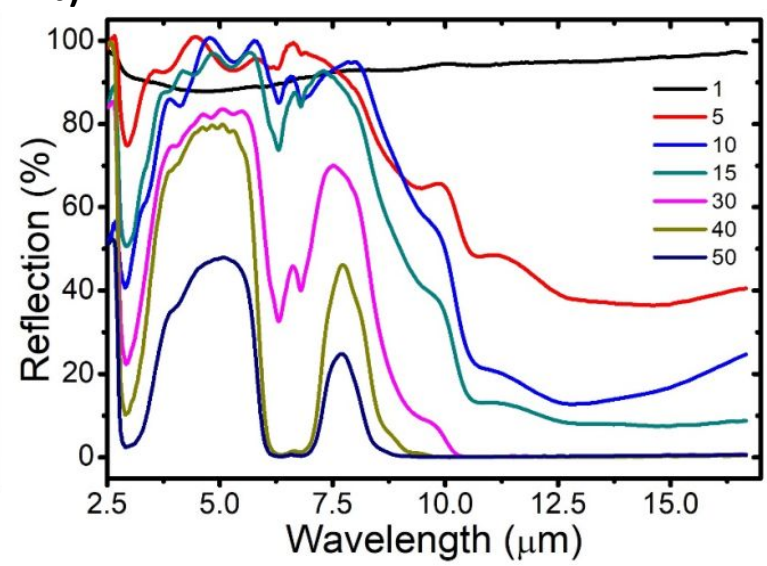

d)

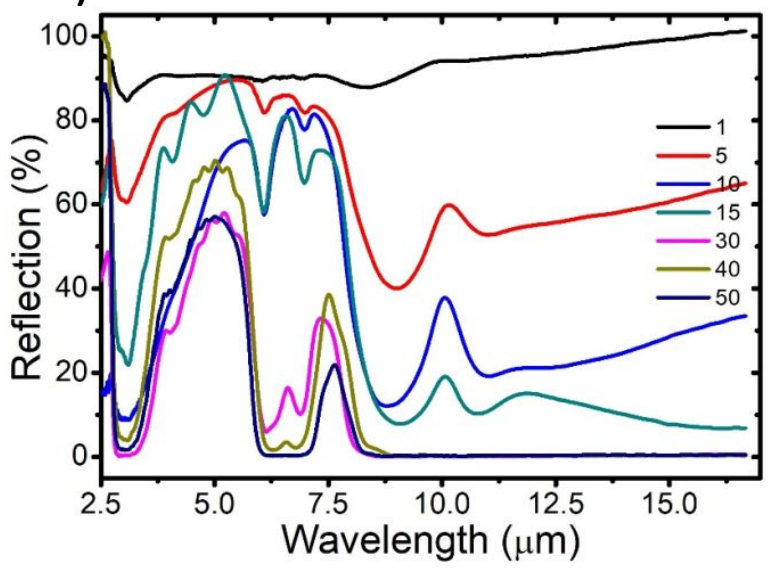

Figure $\mathbf{S}_{\mathbf{1}}$ We measured reflection spectra of ITO coated collapsed nanowire structures. (a), (b) represents measured reflection for DI water dried nanowires and (c), (d) are measured reflection correspondingly for $\mathrm{H}_{3} \mathrm{PO}_{4}$ dried nanowire structures. The inset shows the length of the nanowires (in $\mu \mathrm{m}$ ) corresponding to the color of the lines. 

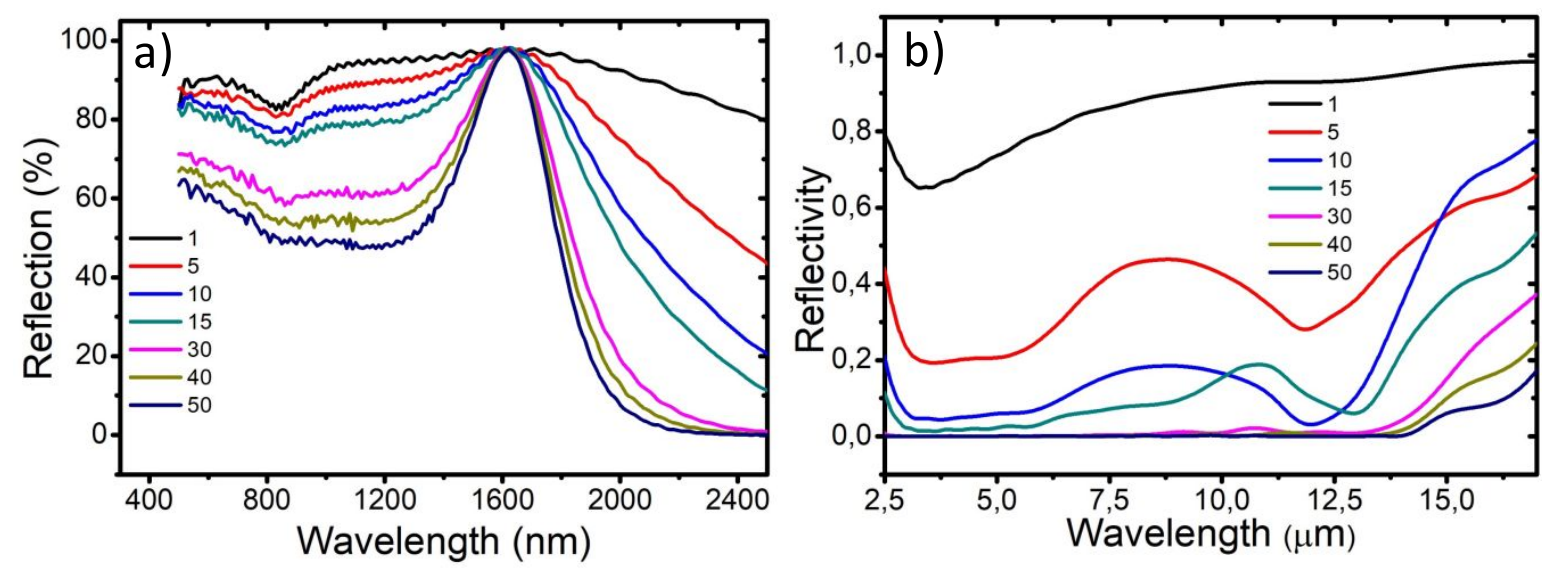

Figure $\mathbf{S}_{\mathbf{2}}$ Figure (a) and (b), represent reflection calculated using FDTD simulation for varying wire lengths of the nanowires. The inset shows the length of the nanowires (in $\mu \mathrm{m}$ ) corresponding to the color of the lines. 